\title{
Dynamical effects in x-ray absorption spectra of graphene and monolayered $h$-BN on Ni(111)
}

\author{
J. Rusz, ${ }^{1,2, *}$ A. B. Preobrajenski, ${ }^{3, \dagger}$ May Ling Ng, ${ }^{3,1}$ N. A. Vinogradov, ${ }^{3,1}$ N. Mårtensson, ${ }^{3,1}$ O. Wessely,, , \\ B. Sanyal, ${ }^{1}$ and O. Eriksson ${ }^{1}$ \\ ${ }^{1}$ Department of Physics and Materials Science, Uppsala University, P.O. Box 530, 75121 Uppsala, Sweden \\ ${ }^{2}$ Institute of Physics, Academy of Sciences of the Czech Republic, Na Slovance 2, CZ-182 21 Prague, Czech Republic \\ ${ }^{3}$ MAX-lab, Lund University, P.O. Box 118, 22100 Lund, Sweden \\ ${ }^{4}$ Department of Mathematics, Imperial College, London SW7 2BZ, United Kingdom
}

(Received 23 November 2009; published 4 February 2010)

\begin{abstract}
We present first-principles calculations of x-ray absorption spectra of graphene and hexagonal BN monolayer on the $\mathrm{Ni}(111)$ substrate. Including dynamical core-hole screening effects according to the theory of Mahan-Nozières-de Dominics (MND) results in an overall good agreement with previously published experimental data and our new observations. This approach provides a unified first-principles description of the electronic structure and core excitations in the $s p^{2}$-bonded materials on metal surfaces and a better insight into the dynamics of screening effects. We demonstrate in particular that the observed spectral features of graphene and hexagonal $\mathrm{BN}$ can be well reproduced with the MND theory, and that they are determined by a delicate balance between initial and final-state effects.
\end{abstract}

DOI: 10.1103/PhysRevB.81.073402

$\mathrm{X}$-ray absorption spectroscopy (XAS) plays an important role in revealing the electronic structure of the $s p^{2}$-bonded layered materials such as graphite and hexagonal boron nitride $(h-\mathrm{BN})$. The element and symmetry selectivity of XAS makes it a convenient tool for probing the B $2 p, \mathrm{C} 2 p$, and $\mathrm{N} 2 p$ density of states (DOS) in $h$-BN (Refs. 1-7) and graphite. ${ }^{8-12}$ However, the influence of the core hole on the spectral shape hampers a straightforward interpretation of the spectra, as was recognized already in the early works. ${ }^{2,3,9,13}$ The near-edge absorption fine structure (NEXAFS) at the $\mathrm{B} 1 s, \mathrm{C} 1 s$, and $\mathrm{N} 1 s$ thresholds in $h$-BN and graphite is affected differently by the corresponding core holes, due to the different core-hole screening. Therefore, a correct theoretical description of all XA spectra in $h$-BN and graphite is difficult from an initial or final-state approach, as well as from a transition state model, because the degree of screening is very different and unknown a priori. Moreover, it can be necessary to assume a co-existence of good and poor screening and to use different theory levels even for one and the same spectrum, e.g., C $1 s$ NEXAFS in bulk graphite. ${ }^{14}$

The problem of a unified theoretical description of the NEXAFS in graphite and $h$-BN is especially severe for the $\pi^{*}$ excitations due to the strong differences in their life time. As shown in Fig. 1, the full width at half maximum of the $\pi^{*}$ resonance (peak A) in bulk materials (black curves) varies from $0.3 \mathrm{eV}$ for the $\mathrm{B} 1 s^{-1} \pi^{*}$ excitation to $1.1 \mathrm{eV}$ for the $\mathrm{C} 1 s^{-1} \pi^{*}$ excitation and further to several $\mathrm{eV}$ for the $\mathrm{N} 1 s^{-1} \pi^{*}$ excitation. Since the symmetry of the potential for the core-excited electron is the same in all three cases, the strong variation in life time of the $\pi^{*}$ excitation is exclusively due to the variation in the electron density on the absorbing site resulting, in particular, in different screening behavior. The differences in the core-hole lifetime account for the different decay dynamics. The relative rate of the nonradiative participator Auger decay is about 30\% for the

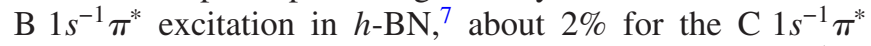
excitation in graphite, ${ }^{11}$ and below $0.5 \%$ for the $\mathrm{N}^{-1} s^{*}$ excitation in $h$-BN, ${ }^{7}$ as determined by resonant photoemission.
PACS number(s): 73.61.Wp, 68.65.Cd, 78.70.Dm, 79.60.Jv

Thus, large variations in the screening characteristics between the $\mathrm{B} 1 s, \mathrm{C} 1 s$, and $\mathrm{N} 1 s$ pre-edge excitations in the $s p^{2}$-bonded materials has hitherto hindered the formulation of a unified theoretical treatment equally suitable for all three situations. In other words, the initial-state, final-state, and dynamical effects contribute to the spectral shape in different and unknown proportions. A well-defined and controlled modification of the electronic structure of graphite and $h$-BN can be easily performed by forming a single atomic layer of these materials on a reactive transition metal surface. The chemisorption of monolayer graphite ( $\mathrm{MG}$ or graphene) and $h$-BN on the lattice-matched and chemically active $\mathrm{Ni}(111)$ substrate is among the most popular interfaces studied in the

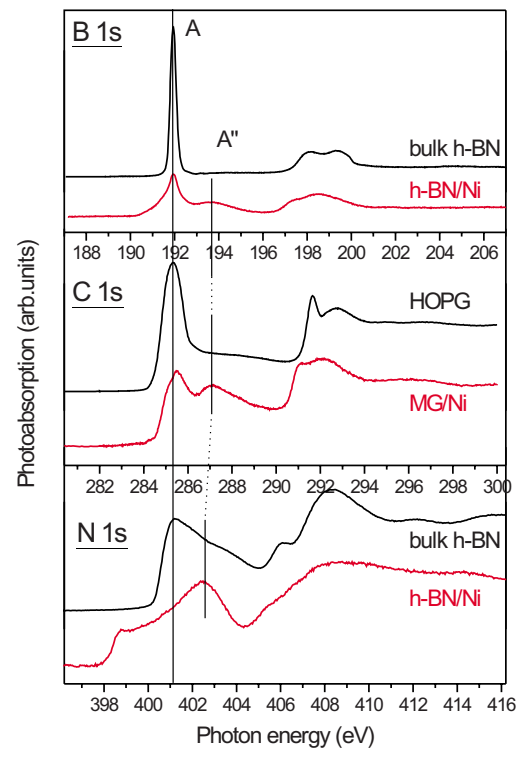

FIG. 1. (Color online) Experimental B $1 s, \mathrm{C} 1 s$, and N $1 s$ NEXAFS spectra from monolayer graphite and $h$-BN adsorbed on $\mathrm{Ni}(111)$ compared with the corresponding spectra of bulk HOPG and $h$-BN. The energy scales are aligned at the position of peak A in the spectra of bulk materials. The angle between the surface normal and the polarization vector of incident radiation is $50^{\circ}$ for all spectra. 
past. The electronic structure of both graphene and $h$-BN on $\mathrm{Ni}(111)$ is perturbed in a similar manner due to the Ni $3 d-\mathrm{MG}\left(h\right.$-BN) $\pi$ orbital mixing. ${ }^{15-18}$

In this paper we systematically treat the process of $\mathrm{x}$-ray absorption in $\mathrm{MG} / \mathrm{Ni}(111)$ and $h-\mathrm{BN} / \mathrm{Ni}(111)$ at the $\mathrm{B} 1 s$, $\mathrm{C} 1 s$, and $\mathrm{N} 1 s$ edges by initial-state, final-state, and dynamical calculations, and compare the results with the B $1 s, \mathrm{C} 1 s$, and N $1 s$ NEXAFS spectra. The experimental data for $h$-BN/Ni(111) were measured previously, ${ }^{17}$ while for the MG/Ni(111) system only the electron energy loss spectra (EELS) were reported so far. ${ }^{19}$ First-principles calculations were performed in the past to explain both EELS in MG/Ni(111), ${ }^{19}$ and NEXAFS spectra in $h$-BN/Ni(111). ${ }^{20} \mathrm{Al}-$ though the agreement with experiment was found to be rather good, only the static effect of the core hole on the spectra could be revealed in these calculations. Here we aim at providing a unified description of the x-ray absorption process in all three cases, and demonstrate that the overall spectral evolution of XAS in graphite and $h$-BN in going from bulk materials to monolayers (MLs) on Ni(111) can be well explained within the Mahan-Nozières-De Dominicis (MND) theory. This theory takes into account the real dynamics of the electronic subsystem in response to the corehole creation, thus providing a more realistic description of the x-ray absorption process than the initial-state or the finalstate calculations. Existing alternative approaches that include the dynamics of the screening processes (based on time-dependent density functional theory or solution of the Bethe-Salpeter equation ${ }^{21}$ ) are computationally rather expensive.

Graphene was grown on $\mathrm{Ni}(111)$ by thermal decomposition of propene gas at $T=950 \mathrm{~K}$. In order to reduce the nucleation rate and the density of grain boundaries we preserved low propene pressure $\left(2-3 \times 10^{-8} \mathrm{mbar}\right)$, and used long preparation time (up to $1 \mathrm{~h}$ ) to ensure saturated coverage. The formation of exactly one graphite ML was checked by the single-component shape of the $\mathrm{C} 1 s$ photoelectron line. If more than $1 \mathrm{ML}$ was grown, a shoulder develops at the low binding energy side of the $\mathrm{C} 1 s$ line. The spectra for $h$-BN/Ni(111) were taken from our earlier work. ${ }^{17}$

All the measured spectra are summarized in Fig. 1. Experimental energy resolution is 40,80 , and $140 \mathrm{meV}$ for $\mathrm{B}$, $\mathrm{C}$, and $\mathrm{N} 1 s$ edges, respectively. Going from $\mathrm{B} 1 s$ to $\mathrm{N} 1 \mathrm{~s}$, peak $\mathrm{A}$ is gradually reduced in intensity, while a new band (A") appears with increasing intensity in all spectra shifted from $\mathrm{A}$ by $1.5-2 \mathrm{eV}$ to higher energies. This effect is rather similar for all spectra, and signifies the interaction with the substrate, since without the substrate, the A" peak disappears. The evolution of new spectral features signifies new chemical bonding situations, and hence provides an excellent means to characterize the interaction with the substrate. However, a good theoretical understanding of these spectral features is needed, and is the focus of this study. Another visible effect is a reduction in the energy separation between the $\pi^{\star}$ and $\sigma^{\star}$ manifolds for all spectra by $0.5-1 \mathrm{eV}$ upon adsorption on $\mathrm{Ni}(111)$, as a result of the lateral bond softening within the adsorbed monolayers.

The calculations of theoretical spectra made use of the MND theory that requires an initial-state electronic structure as well as a final-state electronic structure, in the presence of

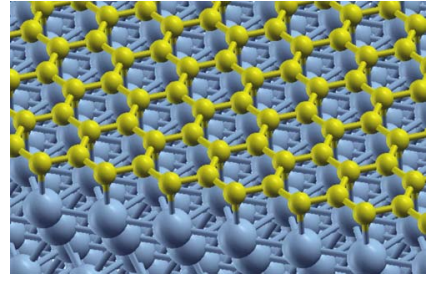

FIG. 2. (Color online) Graphene (or $h$-BN monolayer) on top of the Ni substrate. Note the two nonequivalent positions of carbon atoms: $\mathrm{C}(1)$ on top of the $\mathrm{Ni}$ atom $(\mathrm{N}$ in the case of $\mathrm{BN})$ and $\mathrm{C}(2)$ on top of hollow site (B in the case of $\mathrm{BN}$ ).

a core hole. In our theoretical calculations, which assumed Born-Oppenheimer approximation, we constructed a unit cell consisting of six layers of nickel atoms and a graphene or $h$-BN monolayer, respectively. The vacuum layer was almost $18 \AA$ thick. Both structures were optimized in the ground state, using the VASP $\operatorname{code}^{22,23}$ with the GGA exchangecorrelation potential. ${ }^{24,25}$ The resulting atom positions and interatomic distances agreed well with Refs. 19, 20, and 26, including the placement of nitrogen on top of the nickel and boron on top of the fcc-hollow site, see Fig. 2. For the finalstate calculations (with a core hole) we constructed a $3 \times 3$ $\times 1$ supercell, consisting of 72 atoms. This supercell size turns out to be sufficient for our purposes: the system is metallic and thus the effect of the core hole is well localized around the excited atom core, as documented by negligible (below 0.02) charge transfers on neighboring atoms. All further calculation were then performed by the WIEN2K code ${ }^{27}$ using the optimized structure of Fig. 2. To keep the charge neutrality in the core-hole calculations the missing electron was treated as a homogeneous background charge. This should well reflect the delocalized nature of excited states. Since the XAS reflects the local electronic structure of excited atom thanks to overlap integrals of final $2 p$ states with well-localized initial $1 s$ states, the local Green's function of $\mathrm{B}, \mathrm{C}$, and $\mathrm{N}$ was evaluated within the muffin-tin spheres of radius 1.35 a.u. Due to the limited energy range and sphere radii, the energy-integrated Green's function matrices deviated from the unit matrices. To compensate for this missing charge we performed the following steps for both initial and final-state Green's function: first we diagonalized the energyintegrated Green's function matrix, thus obtaining the transformation matrix from a $|l m s\rangle$ basis into the natural orbital basis. The square roots of the inverse occupation numbers, $1 / \sqrt{n_{i}}$, served as normalization coefficients ( $i$ indexes the natural orbitals). In the second step we transformed all $\langle l m s \mid \mathbf{k} n\rangle\left\langle\mathbf{k} n \mid l^{\prime} m^{\prime} s^{\prime}\right\rangle$ matrices into the natural orbital basis, renormalized their elements by $1 / \sqrt{n_{i} n_{j}}$, and transformed back into the $|l m s\rangle$ basis. After this transformation, the energy-integrated Green's function matrix equals to the unit matrix. These normalized Green's function matrices serve as an input into an efficient MND implementation based on formalism described in Refs. 14 and 28. The energy range of the Green's functions and MND calculation was from -2 Ry up to 3 Ry (zero at Fermi level) with a step of 0.005 Ry and a smearing of 0.01 Ry.

The calculated spectra are summarized in Figs. 3 and 4. In these figures we compare the experimental spectrum (black) 

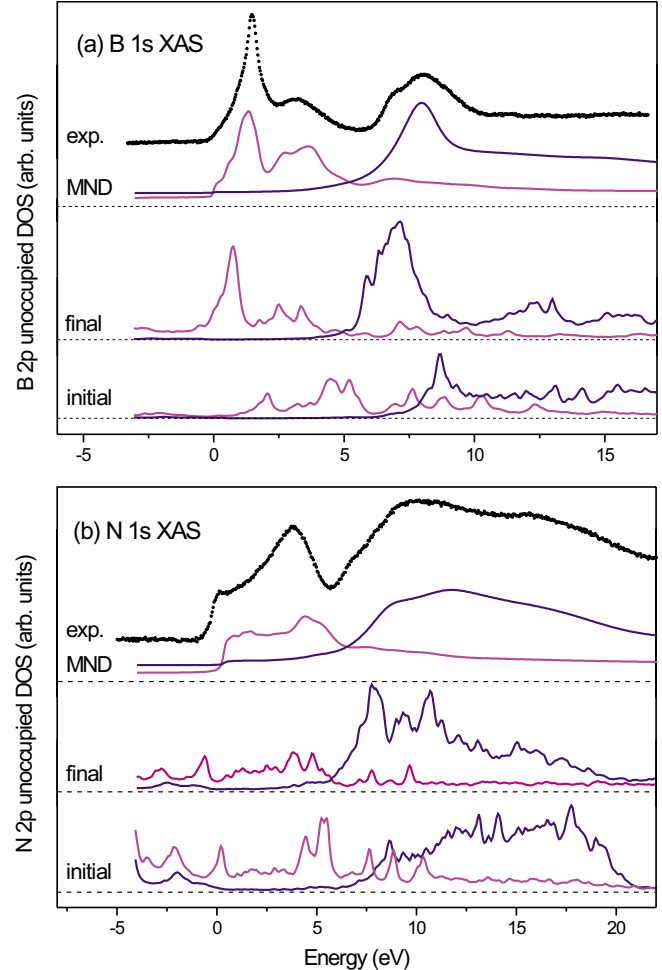

FIG. 3. (Color online) Experimental B $1 s$ (a) and N $1 s$ (b) x-ray absorption spectra from monolayer $h$ - $\mathrm{BN}$ on $\mathrm{Ni}(111)$ compared with the (a) B and (b) N $2 p$ DOS calculated in the ground state ("initial"), in the core-hole state ("final"), and with account for dynamical core-hole screening ("MND"). The DOS is calculated for both $\pi$ states (pink curves) and $\sigma$ states (blue curves).

with the theoretical $\sigma^{\star}$ (blue/dark) and $\pi^{\star}$ (red/light) contributions based on the MND calculation. We also include DOS from the initial-state and final-state electronic structure calculation, for comparison. Because the dipole matrix element within the given energy interval varies less than by $1 \%$, DOS is proportional to the spectrum. Before entering a detailed analysis of the theoretical spectra we observe that the agreement between the experimental spectra and the spectra from MND theory is quite satisfactory, whereas initial-state or final-state theory results in an overall less good agreement. This conclusion was also reached in Refs. 14 and 28 for graphite, carbon nanotubes, and $\mathrm{C}_{60}$.

The case of $h-\mathrm{BN} / \mathrm{Ni}(111)$ is shown in Fig. 3. In the initial-state one can identify a quasigap of the boron $p$-projected DOS, while nitrogen has a peak at the Fermi level. When one $1 s$ electron is removed from the boron core, a strong peak forms just above the Fermi level in the finalstate spectrum. The origin of this peak can be seen in the initial state, at about $2 \mathrm{eV}$ above the Fermi level. Contrary to that, for nitrogen the local $p$-DOS is reduced in the final state due to the more or less simple shift of the $\pi$ DOS by $\sim 1 \mathrm{eV}$ down in the presence of the core hole. This shows that the local electronic structures of boron and nitrogen in $h$-BN/Ni(111) and the response to the creation of a core hole are very different. This stems from different number of valence electrons available for screening of the core hole.

The level of dynamical screening effects, as exemplified by MND calculations of the XAS K-edge fine structures, is
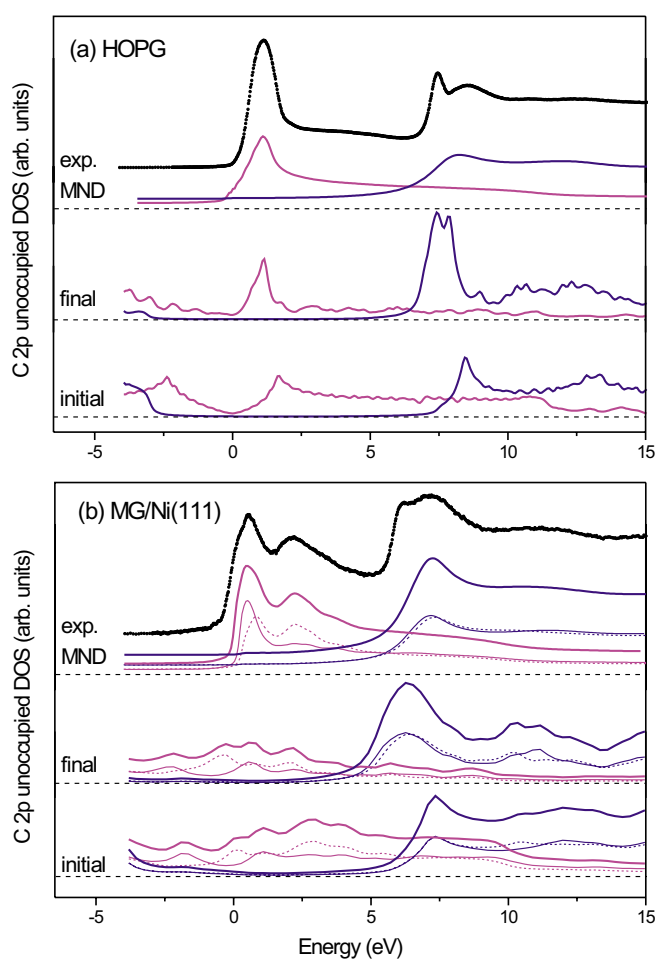

FIG. 4. (Color online) Experimental C 1s NEXAFS from HOPG (a) and MG on $\mathrm{Ni}(111)$ (b) compared with the $\mathrm{C} 2 p$ DOS calculated in the ground state ("initial"), in the core-hole state ("final"), and with account for dynamical core-hole screening ("MND"). The DOS is calculated for both $\pi$ states (pink curves) and $\sigma$ states (blue curves). In the case of MG/Ni(111) the DOS is further separated into the contributions from "on-top" $\mathrm{C}$ atoms (solid curves) and "fcc-hollow" C atoms (dashed curves). Thicker curves represent a sum of contributions from two different types of $\mathrm{C}$ atoms.

consequently also quite different for the $\mathrm{B}$ and $\mathrm{N}$ projected states. In the case of boron [Fig. 3(a)], the MND calculation resembles the final-state calculation. This indicates that the electronic relaxation processes are not sufficient to screen the B $1 s$ core hole due to the lower amount of $p$ electrons on the $\mathrm{B}$ site. The MND calculation somewhat exaggerates the intensity of the peaks at around $3 \mathrm{eV}$ above the edge onset (compared to final-state calculation). On the other hand, a reduction in the $\sigma^{\star}$ component between $7-10 \mathrm{eV}$ in the MND spectra, compared to the final-state spectrum, is in better accord with experiment. On the whole, both final state and MND calculation provide rather good agreement with experiment, indicating that the spectral features are dominated by the final-state electronic structure with a poorly screened core hole.

The situation of the nitrogen edge is more complicated. Neither the initial nor final-state calculation matches the measured spectra. Although the origin of the main experimental structure at $4 \mathrm{eV}$ (the $\pi^{*}$ band shifted upwards by the orbital mixing with the $\mathrm{Ni} 3 d$ states) can be reproduced by both initial-state and final-state calculations, the shape of the $\mathrm{N} 1 s$ absorption onset is clearly incorrect. Besides, the $\sigma^{\star}$ bands are largely dominating the electronic structure at 6-12 $\mathrm{eV}$ and they decay too quickly at higher energies. The situation is dramatically improved by the MND calculation, showing the significance of the dynamical screening pro- 
cesses on the $\mathrm{N} 1 s$ spectral shape. This improvement is facilitated by the gapless nature of the ground-state electronic structure of chemisorbed $h$-BN due to a considerable density of gap states of primarily $\mathrm{N} 2 p\left(\pi^{*}\right)$ character induced by the orbital mixing at the $h$-BN/Ni(111) interface. ${ }^{29}$ For gapless systems the MND approach is known to work especially well, because the core-hole relaxation is crucial there due to the high probability of creating shake-up pairs with low energy.

The case of graphene on $\mathrm{Ni}(111)$ is somewhat complicated by the co-existence of two chemically nonequivalent $\mathrm{C}$ atoms, see Fig. 2, with different number of nearest neighbor Ni atoms. ${ }^{19}$ This causes differences in their local electronic structure, particularly in the $\pi$ DOS. We show in Fig. 4 spectral features projected on both atom types, for the spectra calculated at the initial-state, final-state, and MND theory levels. Neither initial-state nor final-state theory reproduces the experimental curve properly, whereas the MND spectrum provides a significant improvement of the spectral shape. The experimentally observed two-peak structure at the $K$-edge onset is very well reproduced and the MND $\sigma^{\star}$ peak at $7 \mathrm{eV}$ has now a proper relative magnitude (note the large overestimation of its magnitude in both initial and final states). The hybridization-induced peak at $2 \mathrm{eV}$ is present mainly in the MND spectrum originating from the carbon atoms on top of the fcc-hollow sites. These atoms are somewhat closer to the substrate ${ }^{19}$ and their $p_{z}$ orbitals can overlap with the lobes of the Ni $3 d\left(t_{2 g}\right)$ orbitals directed toward these sites. Therefore, despite their larger absolute distance from the nearest $\mathrm{Ni}$ atoms (compared to the $\mathrm{C}$ on top), their local electronic structure is more influenced by the substrate. Note that both initial-state and final-state calculations provide only a qualitative description of these phenomena, while the explicit inclusion of electron correlations into the calculation of transition probabilities results in the correct description of the spectral shape.

In summary, we have succeeded in reproducing all experimental XAS spectra of graphene and $h$-BN monolayers interacting with the $\mathrm{Ni}(111)$ substrate, using a parameter-free first principles method, which explicitly takes into account the dynamics of the core-hole screening. These dynamical screening effects are particularly important for the nitrogen and carbon $K$-edges, due to the high density of the N $2 p$ and C $2 p$ states in the vicinity of the Fermi level. For the boron $K$-edge the MND theory results are similar to those of a more simple static final-state calculation, as a consequence of the poor core-hole screening. In general, our study shows that the real dynamics of the core-hole screening is crucial for correct description of $\mathrm{x}$-ray absorption processes in this class of layered materials and their contacts with substrates. We also show that the new spectral feature, A," is due to a combination of dynamical core-hole screening and the hybridization between the $\pi$ orbitals and the Ni $3 d$ states.

We are grateful for the financial support by the Swedish Foundation for Strategic Research and the Swedish Research Council. Computational resources provided by SNAC.

\footnotetext{
*jan.rusz@fysik.uu.se

†alexeip@maxlab.lu.se

¥Present address: Swedish Nuclear fuel and Waste Management Co,

Low and Intermediate Level Waste, Box 259, 10124 Stockholm, Sweden.

${ }^{1}$ V. A. Fomichev and M. A. Rumsh, J. Phys. Chem. Solids 29, 1015 (1968).

${ }^{2}$ J. Barth, C. Kunz, and T. M. Zimkina, Solid State Commun. 36, 453 (1980).

${ }^{3}$ B. M. Davies, F. Bassani, F. C. Brown, and C. G. Olson, Phys. Rev. B 24, 3537 (1981).

${ }^{4}$ L. J. Terminello et al., J. Vac. Sci. Technol. A 12, 2462 (1994).

${ }^{5}$ D. Li, G. M. Bancroft, and M. E. Fleet, J. Electron Spectrosc. Relat. Phenom. 79, 71 (1996).

${ }^{6}$ R. Franke et al., Chem. Phys. 216, 243 (1997).

${ }^{7}$ A. B. Preobrajenski, A. S. Vinogradov, and N. Mårtensson, J. Electron Spectrosc. Relat. Phenom. 148, 59 (2005).

${ }^{8}$ R. A. Rosenberg, P. J. Love, and V. Rehn, Phys. Rev. B 33, 4034 (1986).

${ }^{9}$ P. E. Batson, Phys. Rev. B 48, 2608 (1993).

${ }^{10}$ P. Skytt, P. Glans, D. C. Mancini, J.-H. Guo, N. Wassdahl, J. Nordgren, and Y. Ma, Phys. Rev. B 50, 10457 (1994).

${ }^{11}$ P. A. Brühwiler, A. J. Maxwell, C. Puglia, A. Nilsson, S. Andersson, and N. Mårtensson, Phys. Rev. Lett. 74, 614 (1995).

${ }^{12}$ R. Ahuja, P. A. Brühwiler, J. M. Wills, B. Johansson, N. Mårtensson, and O. Eriksson, Phys. Rev. B 54, 14396 (1996).

${ }^{13}$ U. von Barth and G. Grossmann, Phys. Scr. 21, 580 (1980).

${ }^{14}$ O. Wessely, M. I. Katsnelson, and O. Eriksson, Phys. Rev. Lett. 94, 167401 (2005).
}

${ }^{15}$ A. Nagashima, N. Tejima, and C. Oshima, Phys. Rev. B 50, 17487 (1994).

${ }^{16}$ E. Rokuta, Y. Hasegawa, K. Suzuki, Y. Gamou, C. Oshima, and A. Nagashima, Phys. Rev. Lett. 79, 4609 (1997).

${ }^{17}$ A. B. Preobrajenski, A. S. Vinogradov, and N. Mårtensson, Phys. Rev. B 70, 165404 (2004).

${ }^{18}$ A. Grüneis and D. V. Vyalikh, Phys. Rev. B 77, 193401 (2008).

${ }^{19}$ G. Bertoni, L. Calmels, A. Altibelli, and V. Serin, Phys. Rev. B 71, 075402 (2005).

${ }^{20}$ R. Laskowski, T. Gallauner, P. Blaha, and K. Schwarz, J. Phys.: Condens. Matter 21, 104210 (2009).

${ }^{21}$ G. Onida, L. Reining, and A. Rubio, Rev. Mod. Phys. 74, 601 (2002).

${ }^{22}$ G. Kresse and J. Hafner, Phys. Rev. B 47, 558 (1993).

${ }^{23}$ G. Kresse and J. Furthmüller, Phys. Rev. B 54, 11169 (1996).

${ }^{24}$ P. E. Blöchl, Phys. Rev. B 50, 17953 (1994).

${ }^{25}$ J. P. Perdew, K. Burke, and M. Ernzerhof, Phys. Rev. Lett. 77, 3865 (1996).

${ }^{26}$ R. Laskowski, P. Blaha, and K. Schwarz, Phys. Rev. B 78, 045409 (2008).

${ }^{27}$ P. Blaha, K. Schwarz, G. K. H. Madsen, D. Kvasnicka, and J. Luitz, WIEN2k, An Augmented Plane Wave+Local Orbitals Program for Calculating Crystal Properties (Techn. Universität Wien, Austria, 2001).

${ }^{28}$ O. Wessely, O. Eriksson, and M. I. Katsnelson, Phys. Rev. B 73, 075402 (2006).

${ }^{29}$ A. B. Preobrajenski, S. A. Krasnikov, A. S. Vinogradov, M. L. Ng, T. Käämbre, A. A. Cafolla, and N. Mårtensson, Phys. Rev. B 77, 085421 (2008). 